pulse is 100 when standing, 96 sitting, but the difference on lying down, when it sinks to 88 , is remarkable, and in accordance with the rule which $I$ have offered.

Look at one more patient, D. H-. I may tell you that his spirometer number is very low, but you see, from his general aspect, his long eyelashes, his gums margined in a way I may more fully describe to you at some future lecture, what you may expect. Under the left clavicle you hear the clicking rhonchus of softened tubercle. His pulse, sitting, is 92 ; standing, 96 .

Examine, by way of contrast, the pulse of T. C-: standing it is 80 ; sitting, even without supporting the back, it soon goes down to 73. The reason is clear to you. This is a patient you have formerly examined, in whom you have detected cardiac disease and pleuritic contraction, but no phthisis.

These instances, taken as it were at random, may suffice to impress an obvious and interesting symptom of the disease. The explanation is not so easy. Is it that the heart, accustomed to a continuous cause of irritation, is less influenced by the trivial excitement which changes of posture might otherwise induce? or is there atrophy of the muscular coat of the arteries, and consequently less variety in the degree of influence exercised by thisstructure on the centre of the circulation? There is one analogy which may suggest a particular line of inquiry, in the fact, particularly observed by Dr. Graves, that the pulse of persons affected with hypertrophy of the heart is remarkably little influenced in its frequency by change of posture. Is the relative weight of the heart to the body generally greater in phthisis than in other diseases? The late Dr. Clendinning investigated this question with much care, and determined it in the affirmative.

The book of post-mortem observations now before you supplies some materials towards the settlement of this question. It records the weights of the hearts of twenty-three men and of nine women. On adding the weights of the former, and dividing by twenty three, I get eight ounces and nine-tenths-of the latter, and dividing by nine, I obtain a result of eight ounces and a third; in both instances, above the average of the healthy heart, and yet many of these subjects were very much emaciated. Should further observations be in harmony with these calculations, there would be so far an analogy between the heart in phthisis and the heart in a state of slight hypertrophy. The fact that the pulse of women and of children is less affected by change of the posture than that of males and adults, affords countenance to the idea that the condition of the muscular coat has an influance in the phenomenon so characteristic of phthisis; whilst the remarkably slight effect produced by emotion on the frequency of the phthisical pulse, lends support to the hypothesis which would assign as an explanation the pre-occupation of the heart with other sources of irritation. The three particulars at which we have glanced may possibly combine their influence, or it may be that the cause has yet to be sought, but of the fact I do not think any substantial doub can be entertained. The hints with which I commenced this lecture may encourage you to expect considerable aid in diagnosis and prognosis from a careful examinanation of the pulse. In this, as in most things, there are two extremes to be avoided. You know that the Chinese were long accustomed to form their diagnosis from the pulse alone, not even seeing the countenance of their fair patients.

Dr. Rucco, in a voluminous work on the subject of the pulse, gives instances in which he predicted the sex of the fotus in utero from the maternal pulse. "We examined," he says, "the pulse of the lady of the Signor Raffaelli, of Naples, and after repeated observations predicted that she would give birth to a male. To this opinion we were led by the difference we observed between the right and left uterine pulse. The first sensibly manifested its organic character, the latter not at all." Dr. Rucco states that " the prediction was verified by the event," and proceeds to give other instances which need not be here adduced, but you may be interested to hear an example in which this physician considers that he detected the influence of a special mental emotion in modifying the pulse. "About three years since, the jaundice attacked a man of letters in consequence of a violent attachment to a young lady, whose parents would not consent to their union. The patient's pulse was low, concentrated, thin, tense, unequal. A small eminence arose between the index and middle fingers, and its smallness and concentration increased at the time the melancholy was deepest, so that it was clear, from the presence of the organic character of the hepatic pulse, that the action of the disappointment or negative he bad received had made a morbose impression upon the vitality of the liver."
You smile at the seeming pretension of this statement, but I would nevertheless advise you to refer to the works of such authors as Rucco; compare their opinions with your own observations, and I am mistaken if you do not find that there is much to be ascertained from certain changes of character in the pulse not susceptible of exact verbal description. Such conditions will aid you in judging of the vital energy in diseases the issue of which may depend more on constitutional peculiarities than in organic change. Independently of the lessened influence of posture, to which it has been one of the objects of this lecture to call your attention, let me repeat that, as a general rule, unnatural quickness of pulse sets in, often at a very early period, in phthisis, and increases with the progress of the disease; that the earlier it is manifested the more, other things being equal, you would apprehend speedy aggravation, but that on the other hand a steady and gradual reduction of the frequency of the pulse would lead you to entertain hopes of important amendment, for under the influence of successful treatment the pulse loses its irritable character and becomes calmer, slower, and fuller. Whilst you would take care not rashly and hastily to pronounce a diagnosis from the state of the pulse alone, you will nevertheless rarely fail to derive considerable assistance from a sagacious regard to its condition.

\section{ON A CASE OF TRUE ANEURISM OF THE HEART.}

BY SAMUEL EDWARDS, M.D.,

PHYSICLAN-ACCOUCHEUR TO THE ST. MARYLEBONE INSTITUTION FoR DISEASES OF WOMEN, ETC. ETC.

Ox May 15th, 1850, I was hastily summoned to the residence of Sir J.S_, who was reported to have suddenly fallen down in a fit whilst entertaining a party of friends. His residence being contiguous to my own, I was by the side of the patient within a few minutes after the occurrence. On entering the drawing-rooms, I found him lying partly on a sofa and two chairs, supported by his friends and servants. His face was livid, eyelids closed, nostrils dilated, mouth open, and tongue slightly protruding, which, with the under lip, was swollen, and of a darker hue than the face generally. The pupils were firmly contracted; not the slightest pulsation could be detected in any of the larger arteries, nor could the hand or ear discover the feeblest action when placed over the præcordial region. After my arrival there were two or three slight apparently spasmodic attempts at inspiration, and on his being moved higher up on the sofa, the pressure on the chest and abdomen occasioned a noise in the throat familiar to those accustomed to post-mortem operations, but which was construed by the friends around to his groaning. Life was, however, extinct. The application of a few external remedies was adopted, not with the idea of proving successful in restoring animation, but merely with the view of passing away a few minutes, in order to prepare his lady and family for the appalling shock which so sudden an event could not fail to produce.

Having obtained permission to examine the chest and abdomen, the following is a brief detail of the post-mortem appear ances:- The external inspection presented no peculiarities; the body was that of a corpulent and well-formed man. On dividing the integuments over the chest and abdomen, there was a subcutaneous stratum of fat, varying from an inch and a quarter to two inches in depth. On removing the sternum, the pericardium was seen considerably distended; on opening it, which was accidentally done by the gentleman assisting me, a considerable quantity of yellowish serum escaped, which, however, was not measured, from this untoward accident. On slitting up the pericardium to a greater extent, a loose, dark clot of blood was seen completely encircling the heart, and consequently quite concealing it. On carefully removing this mass of coagnla, we found it to weigh ten ounces-a weight which was not commensurate with its apparent volume. The heart was lying in its normal situation, and was of natural size. On examining it in situ, to detect if possible the source of the hæmorrhage, we immediately discovered a rent, of about an inch in length, with uneven edges, situated in the centre of the left side of the left ventricle. Around the heart, externally, the investing serous membrane was separated from the muscular substance to at least an inch and a half in diameter. On carrying the investigation deeper, the external rent was found to enter a cavity, formed in the substance of the wall of the left ventricle, which would contain a full-sized walnut; from this a smaller irregular opening, of about a 
quarter of an inch in length, communicated with the cavity of the left ventricle. The muscular tissue of the heart was considerably altered in colour and consistency, presenting the appearance of red softening; it was of a deep claret hue, and under very moderate pressure of the fingers the substance was readily torn; no appearance of anything like pus was discerned. The walls were of normal thickness, and all the valves sound. No other morbid appearances were revealed in the thoracic organs. The abdominal viscera were healthy, with the exception of the kidneys, which were extensively diseased; they were embedded in an enormous quantity of fat, much enlarged, and of a claret colour, or reddish black; both were lobulated as in the foetus. The capsules of each presented ulcerative patches, two of which in the left kidney were very deep, of a conical shape, (bases external,) and extended from a half to three-quarters of an inch into the substance of the organ; the capsules were thickened, and readily torn off; on longitudinal sections of them being made, there was considerable congestion. The tubular portions of both organs were completely obliterated, with the exception of a small portion of the cones in the right kidney.

Remarks.- True aneurism of the heart is a disease of very rare occurrence, and comparatively but few cases have been recorded. Corvisart was the first anthor of importance who described it with accuracy, and althongh Morand and Laennec speak of it, the former confines his observations to valvular aneurisms, and the latter, from his remarks, would lead us to infer that he had not met with true circumseribed ventricular aneurism. This author, in referring to Corvisart's case, terms it "partial dilatation," and further on he says, "I myself have never met with anything of the kind." More recently, Breschat, Cruveilheir, Hope, Latham, Andral, Chassinat, and others, have written on the sulject; but the best monograph, undoubtedly, is that of Mr. Thurnam."

On reference to the above example, it is seen that the morbid change was situated in the left ventricle; such almost invariably has been the case, for on reference to various authors, three cases alone are mentioned as having taken place in the auricles, and even these were doubtful. The reason of its being so uniformly in the left heart, appears explicable from the fact that the arterial tissues are endowed with a higher degree of organization than the venous; because the arterial blood is more stimulating, and thus more liable to create morbid excitement; and because the mechanical exertions of the left ventricle are very considerably greater than the right, and consequently laceration of structure is more likely to occur. The thinness and expansibility of the auricles, the comparatively trifling mechanical power required to expel their contents, are conditions favouring their immunity. Coarctation of their valves, it is true, augments functional power, and gives rise to their dilatation, but then their distention is equal and cannot be called aneurismal. These aneurisms vary greatly in size; the cavity may be not larger than a hazel-nut, or, as in Corvisart's case, it may be as large as that of the heart itself. It may, also, be restricted to the wall of the ventricle, (as in the above instance,) without altering the external configuration; or a tumour may be formed, protruding from the surface, as large as the whole organ. In the report of the post-mortem examination it is seen that the aperture connecting the aneurism with the ventricle was small and irregular. This orifice has been found to differ in its dimensions, in some instances being very small, in others almost equalling the diameter of the cavity itself; its marginsare generally ind urated, either fibro-cartilaginous or osseous. This case, however, proves an exception, its edges being merely irregular and ragged. The lining of the above cavity presented a thin and easily lacerable membrane, which appeared rather a continuation of the endocardium than an adventitious product. When an aneurism of this kind has had its cause in mere unequal dilatation of the walls, the endocardium may be so stretched as to cover unbroken the abnormal cavity; but when the endocardium has been primarily diseased, its corrugated and more opaque condition renders its line of demarcation easily determinable. From the attenuation at the point of rupture it was obvious that life had for some short time been carried on whilst the thin serous investment of the ventricle had alone interposed between the cavity of the tumour and that of the pericardium, and the edges of the laceration were precisely such as would result from a sudden tear. The internal pressure of the current of blood, not being counteracted by organized lamellæ, would continue to produce a gradual absorption of the muscular structure, till at length the attenuated wall of the tumour was unequal to the requisite power of resistance, and the result of rupture super-

\footnotetext{
* Medico-Chirurgical Transactions, vol, xxi., 1838.
}

vened. No put being manifest in the above example, it is quite clear that the pathological change, from this in connexion with other facts, was of a chronic nature; indeed, there are but three cases on record where pus has been noticed in the muscular tissue, and in two out of the three (Latham and Salter) death very rapidly occurred. Inflammation of the endocardium is almost uniformly the concomitant of acute rheumatism, and is assuredly the chief cause of cardiac aneurism. The membrane having become thick and indurated from one of other of the analogous transfurmations, undergoes slight solution of continuity: the fissure becomes wider and wider coagula and bloody detritus are deposited, at first in small quantity, but by exposure to the current of the circulation they increase, until such a volume is accumulated as to exert pressure on the muscular parts adjacent. The same causes and the same effects continuing, irregular dilatation, in other words, aneurism, is the result. There is another change which the organ might undergo--and that was certainly associated with the example now given-which merits mention in speaking of the causes, viz.-softening. It is seen that the substance could be broken beneath the finger, and was abnormally red. The softened portion of the heart may be of different hues,-red, whitish-brown, or yellow. That of the first-named hue, which it is of most importance here to speak of, owing to excess of blood in the capillaries of the muscular and filar mentous tissues, incontestably results from inflammatory de generation. But in acknowledgment of this fact, it is not to be inferred that pus should accompany it as a natural concomitant because every form of inflammation does not necessarily terminate in suppuration. Might it not be caused by primary lesion of the blood, as in purpura and typhus? such changes of the blood might be followed by molecular perversion of the sarcolemma, as we know to be the fact in the more coinmon pathological appearance of fatty degeneration. The kidneys were congested, granular, and lobulated. Congestion and other parenchymatous chang es in these organs are frequent accompaniments of ancient lesion of the heart, especially where hypertrophy has long existed. Dr. Wardell, who has paid much attention to diseases of the heart, and whose extensive experience in pathological anatomy in Edinburgh gives weight to his opinion, states, that organic disease of the left ventricle, more especially in hypertrophy and the various forms of softening, is a very frequent forerunner of morbid changes in the kid. neys. Microscopic investigations have revealed an important fact relative to morbid softening of the viscera, viz.-that whenever the heart is softened, analogous changes may be looked for in the liver and kidneys; and perhaps if attention had been paid to other organs, the same observations would hold good. Lastly, with regard to the diagnosis of cardiac aneurism, no rules can even be attempted; the affection is of so very rare occurrence, and death mostly occurs instantaneously, or from some other form of disease in which auscultation was not indicated. Probably, had the stethoscope been applied, the first sound of the heart wonld have been heard louder, roughened, and more prolonged than ordinary, and this most readily detected at the apex. The valves being healthy, it is fair to infer that the second sound would not be altered. It may be mentioned that about two years ago, the deceased had a slight paralytic affection, manifesting itself in numbness of the left arm: latterly, for several months, he complained of pain ascribed to the posterior part of the thorax, immediately below the point of the scapula. Pathologists have had some difficulty in accounting for neuralgic pains of the heart, but when we remember the experiments on the functions of the pneumogastric nerve, by the late Professor John Reid, the difficulty is immediately removed.

Upper George-street, Bryanstone-square, 1851.

\section{ON A CASE OF HYSTERICAL PARALYSIS. By WILLIAM THORN, L.S.A.}

IN the month of July, 1850, I was requested to meet Mr. Goodchild of Ealing, respecting the case of Mrs. M-, a lady aged 41, married, but without children, who had formerly been a patient of mine while resident in Paddington; she then suffered from disense of the mitral valve of the heart with regurgitation; having a strong bellows murmur and other symptoms of that complaint. Having advised quiet and country air, she removed from town and came under the care of $\mathrm{Mr}$. Goodchild, who had, before I saw her, taken her to a London physician of great eminence; at that time she was hardly able to walk, although not as yet paralysed. I do not know what was then done, except that no examination of the 